\title{
Effects of platelet-rich plasma on mesenchymal stem cells isolated from rat uterus
}

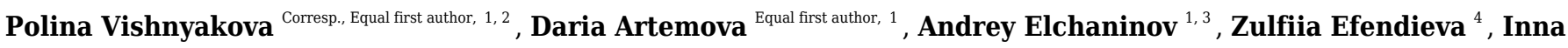 \\ Apolikhina $^{1,4}$, Gennady Sukhikh ${ }^{1,4}$, Timur Fatkhudinov ${ }^{2,5}$ \\ 1 National Medical Research Center for Obstetrics, Gynecology and Perinatology Named after Academician V.I. Kulakov of Ministry of Healthcare of \\ Russian Federation, Moscow, Russia \\ 2 Peoples' Friendship University of Russia (RUDN University), Moscow, Russia \\ 3 Pirogov Russian National Research Medical University (RNRMU), Moscow, Russia \\ 4 I. M. Sechenov First Moscow State Medical University of Ministry of Health of Russia (Sechenov University), Moscow, Russia \\ 5 Scientific Research Institute of Human Morphology, Moscow, Russia \\ Corresponding Author: Polina Vishnyakova \\ Email address: vpa2002@mail.ru
}

Background. Platelet-rich plasma (PRP), which represents a valuable source of growth factors, is increasingly being applied in regenerative medicine. Recent findings suggest the feasibility of using PRP in the treatment of infertility secondary to refractory thin endometrium. Mesenchymal stem/stromal cells (MSCs) of the endometrium are an essential cellular component responsible for extracellular matrix remodeling, angiogenesis, cell-to-cell communication, and postmenstrual tissue repair. Using a rat model, we examine the effects of autologous PRP on MSCs isolated from the uterus and compare them with the effects of autologous ordinary plasma (OP) and complete growth medium.

Methods. MSCs were isolated from uterine tissues via enzymatic disaggregation. Flow cytometry immunophenotyping of the primary cell cultures was complemented by immunocytochemistry for Ki-67 and vimentin. The ability of MSCs to differentiate in osteo-, chondro-, and adipogenic directions was assessed using differentiation-inducing media. The levels of autophagy and apoptosis markers, as well as the levels of matrix metalloproteinase 9 (MMP9) and estrogen receptor $\alpha$, were assessed by western blotting.

Results. After $24 \mathrm{~h}$ incubation, the proliferation index of the PRP-treated MSC cultures was significantly higher than that of the MSC cultures treated with complete growth medium. PRP treatment elevated production of LC3B protein, an autophagy marker, while OP treatment upregulated the expression of stress-induced protein p53 and extracellular enzyme MMP9. The results indicate practical relevance and validity for PRP use in the treatment of infertility. 
1 Effects of platelet-rich plasma on mesenchymal stem 2 cells isolated from rat uterus

3 Vishnyakova Polina 1,2\#*, Artemova Daria ${ }^{1 \#}$, Elchaninov Andrey ${ }^{1,3}$, Efendieva Zulfiia ${ }^{4}$, 4 Apolikhina Inna ${ }^{1,4}$, Sukhikh Gennady ${ }^{1,4}$ and Fatkhudinov Timur ${ }^{2,5}$

5 Author affiliations:

$6{ }^{1}$ National Medical Research Center for Obstetrics, Gynecology and Perinatology Named after 7 Academician V.I. Kulakov of Ministry of Healthcare of Russian Federation, Moscow, Russia

$8 \quad 2$ Peoples' Friendship University of Russia (RUDN University), Moscow, Russia

$9 \quad{ }^{3}$ Pirogov Russian National Research Medical University (RNRMU)

$10{ }^{4}$ I. M. Sechenov First Moscow State Medical University of Ministry of Health of Russia 11 (Sechenov University), Moscow, Russia

${ }^{5}$ Scientific Research Institute of Human Morphology, Moscow, Russia

$$
\text { \#- equal contribution }
$$

\section{Corresponding Author:}

Polina A. Vishnyakova, $\mathrm{PhD}$

Laboratory of regenerative medicine, Research Center for Obstetrics, Gynecology and

Perinatology, Ministry of Healthcare of the Russian Federation, Moscow, Russia. Tel: +79150658577 .

21

Email: vpa2002@mail.ru or vishnyakovapolina@gmail.com

23

\section{Abstract}

Background. Platelet-rich plasma (PRP), which represents a valuable source of growth factors, is increasingly being applied in regenerative medicine. Recent findings suggest the feasibility of using PRP in the treatment of infertility secondary to refractory thin endometrium. Mesenchymal stem/stromal cells (MSCs) of the endometrium are an essential cellular component responsible for extracellular matrix remodeling, angiogenesis, cell-to-cell communication, and postmenstrual tissue repair. Using a rat model, we examine the effects of autologous PRP on MSCs isolated from the uterus and compare them with the effects of autologous ordinary plasma (OP) and complete growth medium. 
Methods. MSCs were isolated from uterine tissues via enzymatic disaggregation. Flow

33

34 cytometry immunophenotyping of the primary cell cultures was complemented by immunocytochemistry for Ki-67 and vimentin. The ability of MSCs to differentiate in osteo-, chondro-, and adipogenic directions was assessed using differentiation-inducing media. The levels of autophagy and apoptosis markers, as well as the levels of matrix metalloproteinase 9 (MMP9) and estrogen receptor $\alpha$, were assessed by western blotting.

Results. After $24 \mathrm{~h}$ incubation, the proliferation index of the PRP-treated MSC cultures was significantly higher than that of the MSC cultures treated with complete growth medium. PRP treatment elevated production of LC3B protein, an autophagy marker, while OP treatment upregulated the expression of stress-induced protein p53 and extracellular enzyme MMP9. The results indicate practical relevance and validity for PRP use in the treatment of infertility.

\section{Introduction}

Platelet-rich plasma (PRP) is a term for collected blood plasma with artificially concentrated platelets (Theoret and Stashak 2014), along with correspondingly increased amounts of latent growth factors as well as other active substances. Blood platelets contain three types of granules: dense granules, $\alpha$-granules, and lysosomes (Flaumenhaft and Sharda 2018); the most abundant are $\alpha$-granules, which contain a number of active substances including chemokines and growth factors (Yun et al. 2016), for example platelet-derived growth factors (PDGFs), transforming growth factors (TGFs), insulin-like growth factors (IGFs), vascular endothelial growth factor (VEGF), epidermal growth factor (EGF), and fibroblast growth factors (FGFs) (Lubkowska, Dolegowska, and Banfi 2012). PRP is also rich in fibrin, fibronectin, and vitronectin (Marx 2019). 
56 Degranulation of platelets upon PRP activation promotes fibrinogen cleavage and formation of

57 the gel-like matrix. The main PRP activators used in laboratory practice are calcium, thrombin,

58 and collagen (Maffulli 2016; Cavallo et al. 2016). The most common PRP activator is calcium,

59 which acts faster than collagen but slower than thrombin (M. H. Kim and Byeon 2019). PRP

60 activation leads to the immediate release of growth factors that start to act at the site of

61 administration. Platelets release $70 \%$ of their total content of growth factors within 10 min of

62 PRP activation with $\mathrm{CaCl}_{2}$, while the remaining $30 \%$ are released over the course of one hour.

63 Moreover, these activated platelets continue to produce extra growth factors. As activated

64 platelets die within approximately $8 \mathrm{~h}$ following the stimulus, PRP activation should be carried

65 out immediately before use (M. H. Kim and Byeon 2019).

66 PRP was introduced as a potential therapeutic for tissue repair by Marx et al. (Marx et al.

67 1998), who reported enhanced rates of bone formation in the osteoplasty of human mandibular

68 defects upon adding PRP to the milled bone graft. Nowadays, PRP is widely used in

69 cosmetology, dentistry, sports medicine, and surgery (Patel et al. 2016; Yuksel et al. 2014;

70 Maffulli 2016); it can be injected in soft tissues, mixed with a graft, layered, sprayed, or used as

71 a biological membrane (Civinini et al. 2011).

72 PRP exerts a local stimulatory effect on cell growth at the site of administration. The benefits

73 of PRP treatment are currently finding recognition in reproductive technologies. A recent study

74 involving 24 female participants with refractory thin endometrium (5 $\mathrm{mm}$ or thinner) or a history

75 of IVF failure demonstrated that a course of three repeated infusions of PRP into the uterine

76 cavity led to significant improvements, with $60 \%$ and $54 \%$ of the patients successfully entering

77 pregnancy and giving birth, respectively (Frantz et al. 2020). The effectiveness of PRP in the

78 treatment of thin endometrium has previously been reported by Zadehmodarres et al.; the study 
79 involved 10 female participants with thin endometrium ( $7 \mathrm{~mm}$ or thinner) who received PRP

80 infusions. After two infusions, endometrial thickness exceeded $7 \mathrm{~mm}$ in all patients, which

81 enabled a frozen-thawed embryo transfer (FET) procedure. As a result, $5(50 \%)$ of the patients

82 successfully entered pregnancy (Zadehmodarres et al. 2017). Coksuer et al. evaluated PRP

83 treatment of thin endometrium as an alternative to estradiol therapy in patients with a history of

84 three or more failed IVF cycles. The resulting average endometrial thicknesses of 10 and $8 \mathrm{~mm}$

85 in the PRP and estradiol groups, respectively, enabled FET in all cases. PRP therapy afforded

86 higher rates of clinical pregnancy and live births (14\% vs 6\%), as well as a lower occurrence of

87 miscarriages ( $3 \%$ versus $6 \%$ ), when compared to estradiol therapy (Coksuer, Akdemir, and Ulas

88 Barut 2019). Additionally, in a study by Kim et al., intrauterine administration of autologous

89 PRP improved implantation and pregnancy outcomes for patients with refractory thin

90 endometrium-associated infertility, indicating profound functional consistency of the effects of

91 PRP (H. Kim et al. 2019). The endometrium is a dynamic structure composed of simple

92 columnar epithelium with uterine glands, as well as the underlying stroma (Pertschuk 1990)

93 containing blood vessels, nerves, collagen, and reticular fibers, along with a variety of stromal

94 cells (Aplin 2018). Isolation of mesenchymal stem/stromal cells (MSCs) from the endometrium

95 (Chan, Schwab, and Gargett 2004; Chan and Gargett 2006) allows the elucidation of tissue-

96 specific functions and markers of these cells, thus opening new prospects for their usage. As

97 actively proliferating cells, MSCs play an important role in tissue homeostasis of the

98 endometrium - they are involved in extracellular matrix remodeling, angiogenesis, cell-to-cell

99 communication, post-menstrual tissue repair, etc. (Mutlu, Hufnagel, and Taylor 2015;

100 Arutyunyan et al. 2016). Endometrial stromal cells are highly susceptible to the action of PRP

101 (Matsumoto et al. 2005); however, only a few works are devoted to studying the exact 
102 mechanisms of PRP action on these cells (Aghajanova et al. 2018). In the perspective of

103 infertility treatment, the use of rat endometrial MSCs forms a good model for studying the action

104 of PRP due to ethical accessibility and sufficient size of the biomaterial, allowing for more cells

105 to be obtained when compared to human pipe endometrial biopsy (Jang et al. 2017).

106 Experimental animal models are accessible and provide better uniformity of samples (Festing 107 and Altman 2002). The use of human biomaterial is invariably associated with heterogeneity of 108 anamneses and preliminary treatment regimens, as well as the use of different collection 109 protocols. Moreover, in the case of thin endometrium, diagnostic curettage is strongly 110 contraindicated, being substituted with pipe biopsies that provide very limited sample volumes 111 that are insufficient for comprehensive examination (Maclean et al. 2020).

112 The uterine tissues were preferred as a source for MSCs over the conventional sources 113 (including red bone marrow, adipose tissue, and the umbilical cord) due to the significant 114 differences in functional properties between MSCs isolated from various locations in the body. 115 For example, MSCs isolated from adipose tissue show higher proliferation rates and a higher 116 adipogenic differentiation capacity compared to MSCs isolated from red bone marrow (Brown et 117 al. 2019). At the same time, bone marrow-derived MSCs are more prone to osteogenic 118 differentiation, whereas MSCs isolated from muscle tissue express myoblastic markers and show 119 the highest rates of differentiation into myogenic progeny (Brown et al. 2019). Despite the 120 common immunophenotype signatures of MSCs isolated from different tissue sources (positive 121 for CD73, CD90, and CD105 expression and negative for the hematopoietic markers CD34 and 122 CD45 on their surface), these cells also exhibit a number of tissue-specific surface markers 123 (Klimczak and Kozlowska 2016) indicating tissue-dependent functional specificity, e.g. SUSD2 
124 and CD146 with co-expression of PDGF-R $\beta$ have been shown to be specific for endometrium

125 (Tempest, Maclean, and Hapangama 2018).

126 The mechanisms of physiological and reparative regeneration of the endometrium are poorly

127 understood. Therefore, we were interested in studying the cellular mechanisms of endometrial

128 regeneration, as well as its stimulation through endometrial MSC activation via PRP. In this

129 study, we examine the effects of autologous PRP on MSCs isolated from rat uterus and compare 130 them with the effects of autologous ordinary plasma (OP).

\section{$131 \quad$ Materials \& Methods}

\section{Ethical disclosure}

The authors state that they have obtained appropriate institutional review board approval or have followed the principles outlined in the Declaration of Helsinki for all human or animal experimental investigations. The study was approved by the Ethical Review Board at the

from the Institute for Bioorganic Chemistry branch animal facilities (Pushchino, Moscow region,

142 Federation, June 1, 2003), and all efforts were made to minimize suffering. The animals were

143 adapted to laboratory conditions $\left(23^{\circ} \mathrm{C}, 12 \mathrm{~h} / 12 \mathrm{~h}\right.$ light/dark, 50\% humidity, ad libitum access to

144 food and water) for 2 weeks prior to experimentation. In adult female rats, estrous cycle stage

145 was determined by taking a vaginal smear. Vaginal smears were stained with methylene blue and

146 stage was determined via assessment of the cellular composition. Following euthanasia in a $\mathrm{CO}_{2}-$

147 chamber at the stage of metestrus, the uterus was dissected and blood was collected via 
148 puncturing the heart. The animals served only as a source of the uteruses and autologous plasma;

149 therefore, no experimental conditions and endpoints were applied.

150 PRP and OP preparation. Blood was collected in tubes containing $2 \mathrm{ml}$ of heparin (5000

$151 \mathrm{IU} / \mathrm{ml}$ ) and $800 \mu \mathrm{l}$ of $10 \%$ sodium citrate. An average of $6 \mathrm{ml}$ of blood was obtained per animal.

152 PRP was obtained based on a protocol developed by Yazigi et al. (Yazigi Junior et al. 2015). The

153 blood was centrifuged at $400 \mathrm{~g}$ for $10 \mathrm{~min}$, the plasma was collected in a new tube and

154 centrifuged again at $400 \mathrm{~g}$ for $10 \mathrm{~min}$ at $4{ }^{\circ} \mathrm{C}$. After centrifugation, $70 \%$ of the supernatant (the

155 platelet-poor plasma, PPP) was discarded. The remaining fraction, the PRP, which belongs to the

156 L type (L-PRP) according to modern classification, was preserved. The platelet counts were

157 determined on a TC20 Automated Cell Counter (Bio-Rad, USA) and constituted $50 \times 10^{6}$

158 platelets $/ \mathrm{ml}$ on average. To obtain OP, the blood was centrifuged at $4{ }^{\circ} \mathrm{C}, 2000 \mathrm{~g}$ for $15 \mathrm{~min}$, and

159 the supernatant was collected. The prepared OP and PRP were aliquoted, frozen, and stored at -

$16020^{\circ} \mathrm{C}$. Before use, PRP was activated by adding $10 \% \mathrm{CaCl}_{2}(10 \mu \mathrm{l}$ per $200 \mu \mathrm{l}$ of PRP) according

161 to the protocol by Messora et al. (Messora et al. 2011).

162 Protocol for obtaining MSCs from rat endometrium. The primary cell cultures were obtained

163 based on the protocol by De Clercq et al. with modifications (De Clercq, Hennes, and Vriens

164 2017). The dissected uterus was minced with scissors in Hank's Balanced Salt Solution (HBBS)

165 and transferred into a tube containing $0.25 \%$ trypsin. The tube was incubated at $4{ }^{\circ} \mathrm{C}$ for $1 \mathrm{~h}$,

166 followed by $1 \mathrm{~h}$ at $22{ }^{\circ} \mathrm{C}$, and, finally, $15 \mathrm{~min}$ at $37^{\circ} \mathrm{C}$ under periodic shaking. The supernatant

167 was taken and the solid bulk was transferred to a solution containing collagenases type I and type

168 IV in $0.05 \%$ trypsin-EDTA solution (collagenase I:collagenase IV:trypsin-EDTA, 1:1:10),

169 incubated at $37^{\circ} \mathrm{C}$ for $30 \mathrm{~min}$, and passed through a $70 \mu \mathrm{m}$ cell strainer. The material remaining

170 on the strainer was transferred to a fresh solution of collagenases with $0.05 \%$ trypsin-EDTA, 
171 incubated at $37^{\circ} \mathrm{C}$ for $15 \mathrm{~min}$, and passed through a strainer again. The resulting suspension,

172 which contained the cells of interest, was centrifuged at $300 \mathrm{~g}$ for $5 \mathrm{~min}$ at $22^{\circ} \mathrm{C}$. Then, the pellet

173 was resuspended in HBBS supplemented with $1 \%$ FBS, centrifuged an additional time, and

174 resuspended in complete growth medium (CGM; DMEM/F-12 supplemented with 10\% FBS, L-

175 glutamine, and penicillin/streptomycin) for cultivation. The obtained cells were verified for

176 compliance with the minimal criteria for MSCs issued by the International Society for Cellular

177 Therapy (Dominici et al. 2006) (adhesion to untreated plastic, specific profile of surface

178 antigens, and in vitro differentiation towards osteogenic, chondrogenic, and adipogenic progeny).

179 The effects of PRP and OP on MSCs were assessed by culturing the cells for $24 \mathrm{~h}$ in medium

180 supplemented with either $10 \%$ PRP or $10 \%$ OP in place of FBS. CGM with $10 \%$ FBS was used

181 as the control.

182 Flow cytometry analysis. Immunophenotyping of the cells for surface and intracellular markers

183 was performed upon reaching $80 \%$ confluence of the cultures. The harvested cells were

184 centrifuged at $800 \mathrm{~g}$ for $10 \mathrm{~min}$, the supernatant was discarded, and then the cells were fixed in

$1852 \%$ paraformaldehyde for $15 \mathrm{~min}$ at room temperature (RT), diluted with $5 \mathrm{ml}$ of PBS, and

186 centrifuged at $1500 \mathrm{~g}$ for $10 \mathrm{~min}$. The pellet was then resuspended in $1 \mathrm{ml}$ of PBS. For

187 immunostaining, $1 \times 10^{5}$ fixed cells were incubated in $100 \mu$ of Rinsing Solution (Miltenyi

188 Biotec, USA) with primary antibodies to CD90 (ab225, 1/100, Abcam), CD45 (130-107-846,

189 clone REA504, 1/20, Miltenyi Biotec), CD105 (ab107595, 1/100, Abcam), and CD34

190 (PAB18289, 1/100, Abnova) at RT for $1 \mathrm{~h}$. Subsequently, the cells were incubated with

191 secondary antibodies: anti-mouse Ig-FITC (ab6785, 1/500, Abcam) or anti-rabbit Ig-PE (sc-

$1923739,1 / 100$, Santa Cruz) at RT for $1 \mathrm{~h}$ in the dark. After the incubation, the cells were washed in 
193 PBS, resuspended in $0.5 \mathrm{ml}$ PBS, and then transferred to fresh tubes for analysis using a

194 FACScan flow cytometer (Becton Dickinson, USA) with CellQuest software.

195 Induced differentiation of MSCs. The ability of MSCs to differentiate in osteo-, chondro-, and 196 adipogenic directions was assessed at early passages (up to 7). The cells were grown to $70 \%$

197 confluency in CGM, and then the growth medium was replaced with differentiation medium (for 198 differentiation) or CGM (for the controls). Differentiation into adipogenic progeny was 199 accomplished using the StemPro ${ }^{\circledR}$ Adipogenesis Differentiation Kit (Gibco) over the course of 7 200 days. At the end of the differentiation process, the cells were fixed with ethyl alcohol:formalin 201 solution (1:4) for $3 \mathrm{~min}$ and then stained with Sudan III (5.7 $\mathrm{mM}$ ) for $10 \mathrm{~min}$, in order to 202 visualize fat droplets. For osteogenic differentiation, the medium was supplemented with 203 dexamethasone $\left(10^{-7} \mathrm{M}\right)$ and ascorbic acid $(0.2 \mathrm{mM})$ over the course of two weeks. At the end of 204 the supplementation period, the cells were fixed with $70 \%$ alcohol. For the detection of 205 mineralization sites, the cells were stained with $40 \mathrm{mM}$ alizarin red $\mathrm{S}$ solution $(\mathrm{pH}=4.7)$ for 5 206 min. Chondrogenic differentiation was performed using the StemPro® Chondrogenesis Differentiation Kit (Gibco). Following two weeks of differentiation, the cells were fixed with $4 \%$ 208 formalin for $1 \mathrm{~h}$. For detection of mucopolysaccharides, the cells were stained with $1 \%$ alcian 209 blue for $24 \mathrm{~h}$. Samples were analyzed using an Axiovert $40 \mathrm{CFL}$ inverted microscope (Zeiss, 210 Germany) using ZEN software (Carl Zeiss, Germany).

211 Immunocytochemistry. The MSCs were grown on glass coverslips (Fisher Scientific) coated 212 with gelatin and placed in Petri dishes $(35 \times 10 \mathrm{~mm})$. For immunocytochemistry, the cells were 213 fixed in $2 \%$ paraformaldehyde. The coverslips with fixed cells were treated with $0.1 \%$ Triton X214100 for 10 min (cell membrane permeabilization) and washed with PBS. Non-specific binding 215 sites were blocked with $1 \%$ BSA in PBS with $0.1 \%$ Tween-20 for 30 min. The coverslips were 
216 incubated with either antibodies to Ki-67 (ab15580, 1/100, Abcam) or vimentin (ab8978, 1/250, 217 Abcam) at $4{ }^{\circ} \mathrm{C}$ for $24 \mathrm{hr}$. After incubation, the coverslips were washed with PBS and then 218 incubated with secondary anti-rabbit-PE antibodies (1/200) or anti-rabbit-FITC antibodies 219 (1/200) in the dark at RT for $1 \mathrm{~h}$ and, subsequently, washed with PBS. To stain the nuclei, the 220 coverslips were incubated with DAPI $(0.004 \mathrm{mg} / \mathrm{ml})$ at $37^{\circ} \mathrm{C}$ for $10 \mathrm{~min}$. Next, the coverslips

221 222 223 224 225 226 227 228 229 230 231 232 233 234 235 236 237 were washed in PBS and mounted in Aqua-Poly Mount (Polysciences, USA). Photographs were taken using a Leica DM 4000B fluorescence microscope (Leica Microsystems, Germany) with LAS AF v.3.1.0 software (Leica Microsystems, Germany).

Western blot assay. The cells were washed with PBS and lysed in ice-cold RIPA buffer. The sample was mixed with $2 \mathrm{X}$ Loading Buffer and incubated at $95^{\circ} \mathrm{C}$ for 1 minute. The samples were stored at $-80{ }^{\circ} \mathrm{C}$ until use and heated for 1 min at $95^{\circ} \mathrm{C}$ before loading. The proteins were separated by $10-12.5 \%$ sodium dodecyl sulfate polyacrylamide gel electrophoresis (SDS-PAGE) and transferred from the gel to PVDF membranes via the semi-wet approach using the TransBlot ${ }^{\circledR}$ Turbo ${ }^{\mathrm{TM}}$ RTA Mini LF PVDF TransferKit (Bio-Rad Laboratories, Inc.). The membranes were blocked with 5\% milk in Tris-buffered saline containing $0.1 \%$ Tween (TTBS) at RT for 1 h, then stained overnight with primary antibodies to LC3B (1:3000, ab48394, Abcam), Bcl-2 (1:1000, ab32124, Abcam), p53 (1:250, ab90363, Abcam), ERa (1:2000, ab3575, Abcam), MMP-9 (1:1000, ab38898, Abcam), and GAPDH (1:500, sc-25778, Santa Cruz). Subsequently, the cells were stained with HRP-conjugated secondary antibodies (1:5000, Bio-Rad Laboratories, Inc.). The chemiluminescent signal was developed using the Novex ECL Kit (Invitrogen, USA) and visualized on a C-DiGit ${ }^{\circledR}$ Blot Scanner (LI-COR, USA) using Image Studio ${ }^{\mathrm{TM}}$ Acquisition Software (LI-COR, USA). A figure of an uncropped membrane is available in the supplementary 
238 information (Fig. S1). Relative protein levels were determined via normalization to GAPDH

239 signals. The bands represent biological replicates (i.e. correspond to different individuals).

240 Statistical analysis. Statistical data processing was performed using GraphPad Prism 8

241 software (GraphPad Software, USA). The Shapiro-Wilk test was applied to assess normality of

242 the distributions. In cases of a normal distribution, one-way ANOVA with Turkey post-hoc test

243 for multiple comparison was used. In cases of a non-normal distribution, the Kruskal-Wallis test

244 with post-hoc Dunn test was used. Differences were considered statistically significant at $245 \mathrm{p}<0.05$.

\section{$246 \quad$ Results}

Characterization of the isolated MSCs. The uterus is a non-classical source of MSCs. Given the wide variability of MSC phenotypes and tissue-specific features (Kwon et al. 2016), the first stage of our study was to demonstrate compliance of our rat uterine mesenchymal cell cultures under the established minimal criteria for MSCs, which includes immunophenotyping and demonstrating the capability of induced in vitro differentiation into different mesenchymal lineages. The obtained cultures of fibroblast-like cells were immunophenotyped using flow forward and side scattering values in an FSC-SSC dot plot diagram (Fig. 1a), which reflects the size and granularity of the cells, respectively. We gated the major pool of single cell events (R1) excluding debris (Fig. 1a). The peaks of fluorescence were distinctly shifted in histograms for the controls stained with only secondary antibodies (Fig. 1b, green curve). We found that $72.7 \%$ of

260 the cells were positive for CD90 and $31.8 \%$ of the cells were positive for CD105, indicating correspondence of the obtained cultures to the CD90+CD105+ phenotype. Interestingly, $3.1 \%$ 
262 and $20.1 \%$ of the cells were positive for CD45 and CD34, respectively. To further confirm the 263 compliance, we stained the cultures for vimentin. The immunocytochemical assay revealed the 264 presence of vimentin protein in $100 \%$ of the cells (Fig. 1c).

265 We promoted differentiation of the cell cultures into adipogenic, osteogenic, and chondrogenic 266 lineages using specific combinations of inducers. The adipogenic differentiation was identified 267 by the formation of lipid droplets, which were revealed by staining with Sudan III (Fig. 1d, left 268 panel). During osteogenic differentiation, the cells formed characteristic conglomerates, with 269 foci of calcification visible following alizarin red staining (Fig. 1d, central panel).

270 Conglomerates were also formed by cells during chondrogenic differentiation, while 271 mucopolysaccharide production was revealed via staining with alcian blue (Fig. 1d, right panel).

272 Effects of autologous PRP and OP on cell proliferation and cell death. Effects of 273 autologous PRP and OP on the rat uterine MSC cultures were evaluated via a $24 \mathrm{~h}$ incubation of 274 the cells in medium containing 10\% PRP or 10\% OP instead of FBS. Accordingly, CGM with $27510 \%$ FBS was used as a control. Proliferation was assessed by immunocytochemical staining for 276 Ki-67 following the incubation (Fig. 2a). The percentage of Ki-67 positive nuclei divided by the 277 total number of nuclei (proliferation index) was $18.1 \%$ for CGM, $41.9 \%$ for PRP, and $21.7 \%$ for 278 OP (Fig. 2b). With regards to PRP treatment, the differences were statistically significant $279(\mathrm{p}=0.04)$.

280 To evaluate the activation or inhibition of apoptosis, we analyzed the production of the stress281 induced protein $\mathrm{p} 53$ and the anti-apoptotic protein Bcl-2. p53 protein levels were significantly 282 higher in the cells exposed to OP $(p=0.03)$ when compared with control (Fig. 2c, d). After PRP 283 exposure, the $\mathrm{p} 53$ production level did not change. Relative levels of Bcl-2 production did not 284 differ among the studied groups (Fig. 2c, e). 
Autophagy was assessed by analyzing the level of production of the autophagy marker LC3B.

286

287

288

289

290

291

292

293

294

295

296

297

298

299

300

301

302

303

304

305

306

307

Western blot analysis revealed elevated production of LC3B protein in the cells exposed to autologous PRP when compared with control (CGM, Fig. 2c, f); the observed effect was statistically significant.

\section{Endometrium receptivity: effects of autologous PRP and OP on matrix metalloproteinase}

9 (MMP9) and estrogen receptor $\boldsymbol{\alpha}(\mathbf{E R \alpha})$ production. Zinc-metalloproteinase MMP9

participates in extracellular matrix remodeling. We estimated potential invasiveness of MSCs as a correlate of MMP9 production. Although elevated levels of MMP9 production were observed in both the OP and PRP groups when compared to CGM, the MMP9 upregulation was only statistically significant for the OP treated cultures $(p=0.03$, Fig. 3a, b). Additionally, we compared ER $\alpha$ levels, which can be partially associated with endometrial receptivity (Fig. 3c, d). Despite elevated ER $\alpha$ levels in the PRP and OP treated cultures, these differences were not significant.

\section{Discussion}

The administration of PRP for treatment of thin endometrium has been already introduced into clinical practice in a pilot study by Kim et al., which aimed at increasing endometrial thickness and, accordingly, the probability of implantation (H. Kim et al. 2019). However, the exact mechanism(s) behind the positive effect of PRP on endometrial thickness remains obscure. In this study, we attempt to identify signaling pathways activated in the multipotent stromal cells of the endometrium under the influence of autologous PRP using rat endometrium as a model. Endometrial MSCs play a key role in the stroma: they participate in the regeneration of the functional layer of the endometrium due to the presence of sex hormone receptors and their ability for extracellular matrix remodeling (Mutlu, Hufnagel, and Taylor 2015). These features 
308 emphasize the importance of MSCs in determining receptivity of the endometrium during the 309 implantation period. We isolated primary cultures of stromal cells from rat uterus and proved 310 that these cells were essentially MSCs due to their ability to differentiate towards adipogenic, 311 osteogenic, and chondrogenic lineages in vitro. Phenotypic profiles of the isolated primary 312 cultures were compliant with the CD90+CD105+Vimentin+CD45-CD34- profiles established for 313 MSCs. The presence of cells positive for the CD45 and CD34 markers can be explained by a 314 small admixture of hematopoietic cells and high vascularization of the endometrial stroma. 315 Considering the precedent of $81 \%$ CD34- for synovial fluid-derived MSCs and 97.5\% CD34- for 316 synovial membrane-derived MSCs cultures classified as CD34 negative, we classify the obtained 317 cultures as CD45 and CD34 negative. The percentage of cells positive for CD105 was lower than 318 expected; however, the published evidence indicates substantial variability of this parameter 319 when assessing MSCs isolated from various tissue sources (Ponnaiyan and Jegadeesan 2014). In 320 addition, it was shown that the population of MSCs isolated from one source is heterogeneous 321 and can be represented by both CD105- and CD105+ cells (Pham, Vu, and Van Pham 2019). It is 322 important to note that CD105 is a component of the TGF $\beta$ receptor, whose level fluctuates 323 depending on the estrous cycle in the rat (Caron et al. 2009).

324 In the experiments involving plasma treatment, the obtained MSCs were incubated for $24 \mathrm{~h}$ in 325 control medium (DMEM/F-12 based CGM with 10\% FBS) or medium supplemented with 326 autologous $10 \%$ PRP or 10\% OP instead of FBS. We observed increased proliferation rates of 327 MSCs under the influence of PRP compared with the influence of FBS. This observation 328 indicates mitogenic effects of PRP on stromal cells. We also studied the protein production 329 levels for the established markers of apoptosis and autophagy. The stress-induced protein p53 is 330 a transcription factor that regulates the cell cycle and acts as a tumor suppressor (Labuschagne, 
331 Zani, and Vousden 2018). An important function of p53 is to prevent the accumulation of DNA

332 damage. In the case of damage to cellular DNA, p53 promotes cell cycle arrest and triggers the

333 emergency DNA repair systems; in the case of extensive DNA damage, p53 triggers apoptosis

334 (Wang, Simpson, and Brown 2015). We show that OP promotes increased production of p53

335 protein by stromal cells, revealing a certain degree of cellular stress associated with the OP

336 treatment. At the same time, both PRP and OP have no effect on the levels of production of the 337 anti-apoptotic Bcl-2 protein by MSCs. Bcl-2 is an anti-apoptotic protein localized on the outer

338 mitochondrial membrane, as well as the membranes of the nuclear envelope and the endoplasmic

339 reticulum (Delbridge et al. 2016). Apparently, Bcl-2 activation is not involved in the effects of

340 PRP and OP.

341 We used LC3B, a protein which is involved in the biogenesis of autophagosomes (Barth, 342 Glick, and Macleod 2010), as an autophagy marker. Upon binding to the membrane, LC3

343 conjugates with phosphatidylethanolamine lipid (Tanida, Ueno, and Kominami 2008). Following 344 autophagosome formation, LC3-II is released into the cytosol from the outer layer, while LC3-II

345 located in the inner layer is exposed to hydrolases. LC3B-II is conventionally used as a marker

346 of autophagosomal activity. We observed excessive production of LC3B in MSCs after exposure

347 to PRP. This observation suggests that PRP contributes to self-renewal of the endometrial

348 stromal cells by enhancing autophagy. Rapamycin-induced autophagy was shown to enhance the 349 viability of MSCs, while shRNA-mediated knockdown of autophagy-associated genes decreased 350 their viability (Molaei et al. 2015; Jakovljevic et al. 2018). Decreased autophagy levels are

351 currently considered to be one of the mechanisms underlying the aging of MSCs (Fafián-Labora,

352 Morente-López, and Arufe 2019). 
Endometrial receptivity is the result of a combination of different characteristics of the

354

355

356

357

358

359

360

361

362

363

364

365

366

367

368

369

370

371 372 trophoblast invasion (Bischof, Meisser, and Campana 2002). In this study, we used MMP9 373 production as an indicator of the MSCs capacity for extracellular matrix remodeling, which is an 374 important parameter of morphogenetic plasticity and receptivity of the endometrium. We

endometrium that are responsible for its ability to promote implantation. One indicator of endometrial receptivity is the level of ER $\alpha$, as this receptor mediates the actions of estrogens preparing the endometrium for implantation via increasing its thickness through increasing the cell proliferation rate. In clinical practice, the level of ER $\alpha$ production can be evaluated by staining the endometrial cells with antibodies to ER $\alpha$ (Glasser et al. 2002), which is expressed by several subpopulations of endometrial stromal cells, including MSCs (Zhou et al. 2001). Therefore, we employed ER $\alpha$ as a marker to reflect the conditional receptivity of the endometrium in our model. We observed no significant differences in the level of ER $\alpha$ production upon exposure of the MSCs to PRP or OP, which may indicate that the beneficial effects of these agents are estrogen-independent. However, this finding should be verified on larger samples to exclude individual variations in the estrogen dependence of the plasma treatment effects.

Aside from being sensitive to the action of hormones, the endometrium should be susceptible and supportive to invasion by the trophoblast. Trophoblast cells bind to the endometrium, proliferate, and penetrate deep into the stroma where they come into contact with maternal blood. Progressive remodeling of the extracellular matrix is a hallmark of this process (Bischof, Meisser, and Campana 2002). The expression of matrix metalloproteinases, observed not only by the trophoblast but also by the endometrial stromal cells, provides effective support to the 
375 demonstrated an increase in MMP9 production specifically facilitated by OP treatment when

376 compared to CGM.

377 In general, the obtained results indicate stimulatory effects by PRP on the endometrium, as

378 indicated by the behavior of the MSCs isolated from it. Other studies have also indicated that

379 PRP enhances cell proliferation and differentiation in the uterus (Etulain et al. 2018; Aghajanova

380 et al. 2018). Intrauterine administration of PRP to rats with damaged endometrium enhanced

381 proliferation, thus promoting tissue repair and also reducing fibrosis (Jang et al. 2017).

382 In a study by Kim and et al., therapeutic effects from PRP treatment in a mouse model of

383 Asherman's syndrome were demonstrated (J. H. Kim et al. 2020). Asherman's syndrome is

384 characterized by formation of adhesions and fibrotic lesions inside the uterus. Injections of

385 human PRP at the sites of damage reduced the degree of fibrosis and promoted recovery. It was

386 shown that, after PRP therapy, implantation potential substantially increased and $83.3 \%$ of PRP-

387 treated mice gave birth to live offspring, compared with $0.0 \%$ in the control group.

388 The receptivity of the endometrium strongly depends on the inflammatory status and favorable 389 antimicrobial conditions. Pronounced anti-inflammatory effects of PRP on the endometrium 390 were demonstrated in horses by Reghini et al. (Reghini et al. 2016). Uterine infusions of PRP to 391 mares with chronic endometritis significantly reduced the signs of neutrophilic infiltration and 392 the volume of intrauterine fluid accumulation when observed $24 \mathrm{~h}$ after treatment (Reghini et al. 393 2016). The mechanisms of PRP action on the endometrial cells are still disputable and, 394 apparently, involve anti-inflammatory and pro-proliferative signaling.

395 The effects of OP on the endometrium are notably understudied. In our experiments, OP 396 induced up-regulation of p53 and MMP9 protein expression, so the beneficial effects of OP are

397 less pronounced. The growth factors released from the PRP activated platelets stimulate 
398 proliferation of endometrial cells and enhance autophagy, indicating advantages of PRP as a

399 treatment agent for endometrial dysfunction.

400 Conclusions

401 The use of modified blood plasma is expanding in clinical applications. This study evaluates

402 the effects of autologous PRP and OP on tissue-specific MSCs from rat endometrium. Exposure

403 to PRP enhances proliferation of the uterine MSCs, with a significant increase in autophagy.

404 Exposure to OP increased production of the stress-induced protein p53 and the extracellular

405 enzyme MMP9. The results indicate the potency of PRP for the treatment of infertility,

406 particularly in the management of thin endometrium. An understanding of the molecular

407 pathways mediating the beneficial effects of PRP will expand the range of PRP applications.

408

409 Acknowledgements

410 Special thanks to Natalia Usman for her help with proofreading of the manuscript.

411 References

412 Aghajanova, Lusine, Sahar Houshdaran, Shaina Balayan, Evelina Manvelyan, Juan C. Irwin,

413 Heather G. Huddleston, and Linda C. Giudice. 2018. "In Vitro Evidence That Platelet-Rich

$414 \quad$ Plasma Stimulates Cellular Processes Involved in Endometrial Regeneration.” Journal of

415 Assisted Reproduction and Genetics 35 (5): 757-70. https://doi.org/10.1007/s10815-018-

$416 \quad 1130-8$.

417 Aplin, John. 2018. “Uterus-Endometrium.” In Encyclopedia of Reproduction, 326-32. Elsevier. 418 https://doi.org/10.1016/B978-0-12-801238-3.64654-8.

419 Arutyunyan, I. V., T. H. Fatkhudinov, A. V. El'chaninov, A. V. Makarov, E. Yu Kananykhina,

420 N. Yu Usman, E. Sh Raimova, D. V. Goldshtein, and G. B. Bol'shakova. 2016. "Effect of

421 Endothelial Cells on Angiogenic Properties of Multipotent Stromal Cells from the 
422 Umbilical Cord during Angiogenesis Modeling in the Basement Membrane Matrix.”

423 Bulletin of Experimental Biology and Medicine 160 (4): 575-82.

$424 \quad$ https://doi.org/10.1007/s10517-016-3221-9.

425 Barth, Sandra, Danielle Glick, and Kay F Macleod. 2010. “Autophagy: Assays and Artifacts.”

426 The Journal of Pathology 221 (2): 117-24. https://doi.org/10.1002/path.2694.

427 Bischof, P., A. Meisser, and A. Campana. 2002. "Control of MMP-9 Expression at the Maternal-

428 Fetal Interface.” In Journal of Reproductive Immunology, 55:3-10. Elsevier.

429 https://doi.org/10.1016/S0165-0378(01)00142-5.

430 Brown, Christina, Christina McKee, Shreeya Bakshi, Keegan Walker, Eryk Hakman, Sophia

431 Halassy, David Svinarich, Robert Dodds, Chhabi K. Govind, and G. Rasul Chaudhry. 2019.

432 "Mesenchymal Stem Cells: Cell Therapy and Regeneration Potential.” Journal of Tissue

$433 \quad$ Engineering and Regenerative Medicine 13 (9): 1738-55.

434 https://doi.org/10.1002/term.2914.

435 Caron, Pierre Luc, Guylaine Fréchette-Frigon, Carl Shooner, Valérie Leblanc, and Eric Asselin.

436 2009. "Transforming Growth Factor Beta Isoforms Regulation of Akt Activity and XIAP

437 Levels in Rat Endometrium during Estrous Cycle, in a Model of Pseudopregnancy and in

438 Cultured Decidual Cells.” Reproductive Biology and Endocrinology 7 (August).

439 https://doi.org/10.1186/1477-7827-7-80.

440 Cavallo, Carola, Alice Roffi, Brunella Grigolo, Erminia Mariani, Loredana Pratelli, Giulia Merli,

441 Elizaveta Kon, Maurilio Marcacci, and Giuseppe Filardo. 2016. "Platelet-Rich Plasma: The

442 Choice of Activation Method Affects the Release of Bioactive Molecules.” BioMed

443 Research International 2016. https://doi.org/10.1155/2016/6591717.

444 Chan, Rachel W.S., and Caroline E. Gargett. 2006. "Identification of Label-Retaining Cells in 
445 Mouse Endometrium.” STEM CELLS 24 (6): 1529-38.

$446 \quad$ https://doi.org/10.1634/stemcells.2005-0411.

447 Chan, Rachel W.S., Kjiana E. Schwab, and Caroline E. Gargett. 2004. "Clonogenicity of Human 448 Endometrial Epithelial and Stromal Cells1.” Biology of Reproduction 70 (6): 1738-50.

449 https://doi.org/10.1095/biolreprod.103.024109.

450 Civinini, Roberto, Armando Macera, Lorenzo Nistri, Birgit Redl, and Massimo Innocenti. 2011.

451 "The Use of Autologous Blood-Derived Growth Factors in Bone Regeneration.” Clinical

$452 \quad$ Cases in Mineral and Bone Metabolism. CIC Edizioni Internazionali.

453 Clercq, Katrien De, Aurélie Hennes, and Joris Vriens. 2017. "Isolation of Mouse Endometrial

454 Epithelial and Stromal Cells for in Vitro Decidualization.” Journal of Visualized

455 Experiments 2017 (121). https://doi.org/10.3791/55168.

456 Coksuer, Hakan, Yesim Akdemir, and Mert Ulas Barut. 2019. "Improved in Vitro Fertilization

457 Success and Pregnancy Outcome with Autologous Platelet-Rich Plasma Treatment in

$458 \quad$ Unexplained Infertility Patients That Had Repeated Implantation Failure History.”

$459 \quad$ Gynecological Endocrinology 35 (9): 815-18.

$460 \quad$ https://doi.org/10.1080/09513590.2019.1597344.

461 Delbridge, Alex R.D., Stephanie Grabow, Andreas Strasser, and David L. Vaux. 2016. "Thirty

462 Years of BCL-2: Translating Cell Death Discoveries into Novel Cancer Therapies.” Nature

463 Reviews Cancer. Nature Publishing Group. https://doi.org/10.1038/nrc.2015.17.

464 Dominici, M., K. Le Blanc, I. Mueller, I. Slaper-Cortenbach, F. C. Marini, D. S. Krause, R. J.

465 Deans, A. Keating, D. J. Prockop, and E. M. Horwitz. 2006. "Minimal Criteria for Defining

466 Multipotent Mesenchymal Stromal Cells. The International Society for Cellular Therapy

467 Position Statement.” Cytotherapy 8 (4): 315-17. 
https://doi.org/10.1080/14653240600855905.

469 Etulain, Julia, Hebe A. Mena, Roberto P. Meiss, Gustavo Frechtel, Susana Gutt, Soledad

470 Negrotto, and Mirta Schattner. 2018. “An Optimised Protocol for Platelet-Rich Plasma

471 Preparation to Improve Its Angiogenic and Regenerative Properties." Scientific Reports 8

472 (1): 1-15. https://doi.org/10.1038/s41598-018-19419-6.

473 Fafián-Labora, Juan Antonio, Miriam Morente-López, and María C. Arufe. 2019. "Effect of 474 Aging on Behaviour of Mesenchymal Stem Cells.” World Journal of Stem Cells 11 (6):

475 337-46. https://doi.org/10.4252/wjsc.v11.i6.337.

476 Festing, Michael F.W., and Douglas G. Altman. 2002. "Guidelines for the Design and Statistical

477 Analysis of Experiments Using Laboratory Animals.” ILAR Journal 43 (4): 244-57.

$478 \quad$ https://doi.org/10.1093/ilar.43.4.244.

479 Flaumenhaft, Robert, and Anish Sharda. 2018. "The Life Cycle of Platelet Granules."

480 F1000Research. Faculty of 1000 Ltd. https://doi.org/10.12688/f1000research.13283.1.

481 Frantz, Nilo, Marcelo Ferreira, Marcos Iuri Kulmann, Gerta Frantz, Adriana Bos-Mikich, and

482 Ricardo Oliveira. 2020. "Platelet-Rich Plasma as an Effective Alternative Approach for

483 Improving Endometrial Receptivity - a Clinical Retrospective Study.” JBRA Assisted

484 Reproduction. https://doi.org/10.5935/1518-0557.20200026.

485 Glasser, Stanley R., John D. Aplin, Linda C. Giudice, and Siamak Tabibzadeh. 2002. The

486 Endometrium. Taylor \& Francis.

487 Jakovljevic, Jelena, C. Randall Harrell, Crissy Fellabaum, Aleksandar Arsenijevic, Nemanja

488 Jovicic, and Vladislav Volarevic. 2018. "Modulation of Autophagy as New Approach in

489 Mesenchymal Stem Cell-Based Therapy.” Biomedicine and Pharmacotherapy. Elsevier

$490 \quad$ Masson SAS. https://doi.org/10.1016/j.biopha.2018.05.061. 
491 Jang, Hang Yong, Soo Min Myoung, Jeong Min Choe, Tak Kim, Yong Pil Cheon, Yong Min 492 Kim, and Hyuntae Park. 2017. "Effects of Autologous Platelet-Rich Plasma on

493

494

495

496

497

498

499

500

501

502

503

504

505

506

507

508

509

510

511

512

513

Kim, Hounyoung, Ji Eun Shin, Hwa Seon Koo, Hwang Kwon, Dong Hee Choi, and Ji Hyang Regeneration of Damaged Endometrium in Female Rats.” Yonsei Medical Journal 58 (6): 1195-1203. https://doi.org/10.3349/ymj.2017.58.6.1195. Kim. 2019. "Effect of Autologous Platelet-Rich Plasma Treatment on Refractory Thin Endometrium during the Frozen Embryo Transfer Cycle: A Pilot Study." Frontiers in Endocrinology 10 (FEB). https://doi.org/10.3389/fendo.2019.00061.

Kim, Ji Hye, Mira Park, Jin Young Paek, Woo Sik Lee, Haengseok Song, and Sang Woo Lyu. 2020. “Intrauterine Infusion of Human Platelet-Rich Plasma Improves Endometrial Regeneration and Pregnancy Outcomes in a Murine Model of Asherman's Syndrome.” Frontiers in Physiology. https://doi.org/10.3389/fphys.2020.00105.

Kim, Moon Hee, and Hyeon Seob Byeon. 2019. "Review for Good Platelet-Rich Plasma Procedure in Cosmetic Dermatology and Surgery." Journal of Cosmetic Medicine 3 (1): 113. https://doi.org/10.25056/jcm.2019.3.1.1.

Klimczak, Aleksandra, and Urszula Kozlowska. 2016. "Mesenchymal Stromal Cells and TissueSpecific Progenitor Cells: Their Role in Tissue Homeostasis.” Stem Cells International. https://doi.org/10.1155/2016/4285215.

Kwon, Ahlm, Yonggoo Kim, Myungshin Kim, Jiyeon Kim, Hayoung Choi, Dong Wook Jekarl, Seungok Lee, Jung Min Kim, Jong Chul Shin, and In Yang Park. 2016. “Tissue-Specific Differentiation Potency of Mesenchymal Stromal Cells from Perinatal Tissues.” Scientific Reports. https://doi.org/10.1038/srep23544. 
514

515

516

517

518

519

520

521

522

523

524

525

526

527

528

529

530

531

532

533

534

535

536

by P53 - Cancer and Beyond." Biochimica et Biophysica Acta-Reviews on Cancer.

Elsevier B.V. https://doi.org/10.1016/j.bbcan.2018.06.001.

Lubkowska, A., B. Dolegowska, and G. Banfi. 2012. "Growth Factor Content in PRP and Their Applicability in Medicine.” Journal of Biological Regulators and Homeostatic Agents.

Maclean, Alison, Areege Kamal, Meera Adishesh, Rafah Alnafakh, Nicola Tempest, and Dharani K. Hapangama. 2020. “Human Uterine Biopsy: Research Value and Common Pitfalls.” International Journal of Reproductive Medicine 2020 (April): 1-7. https://doi.org/10.1155/2020/9275360.

Maffulli, Nicola. 2016. Platelet Rich Plasma in Musculoskeletal Practice. Platelet Rich Plasma in Musculoskeletal Practice. https://doi.org/10.1007/978-1-4471-7271-0.

Marx, Robert E. 2019. “In Situ Tissue Engineering.” In Regenerative Strategies for Maxillary and Mandibular Reconstruction, edited by James Melville, Jonathan Shum, Simon Young, and Mark Wong, 73-86. Springer International Publishing. https://doi.org/10.1007/978-3319-93668-0_7.

Marx, Robert E., Eric R. Carlson, Ralph M. Eichstaedt, Steven R. Schimmele, James E. Strauss, and Karen R. Georgeff. 1998. "Platelet-Rich Plasma: Growth Factor Enhancement for Bone Grafts.” Oral Surgery, Oral Medicine, Oral Pathology, Oral Radiology, and Endodontics. https://doi.org/10.1016/S1079-2104(98)90029-4.

Matsumoto, Harunobu, Kaei Nasu, Masakazu Nishida, Hiroko Ito, Sun Bing, and Isao Miyakawa. 2005. "Regulation of Proliferation, Motility, and Contractility of Human Endometrial Stromal Cells by Platelet-Derived Growth Factor." The Journal of Clinical Endocrinology \& Metabolism 90 (6): 3560-67. https://doi.org/10.1210/jc.2004-1918.

Messora, Michel Reis, Maria José Hitomi Nagata, Flávia Aparecida Chaves Furlaneto, Rita 
Cássia Menegati Dornelles4, Suely Regina Mogami Bomfim, Tatiana Miranda Deliberador, Valdir Gouveia Garcia, and Alvaro Francisco Bosco. 2011. “A Standardized Research Protocol for Platelet- Rich Plasma (PRP) Preparation in Rats." RSBO Revista Sul-Brasileira de Odontologia.

541 Molaei, Sedigheh, Mehryar Habibi Roudkenar, Fatemeh Amiri, Mozhgan Dehghan Harati, 542 Marzie Bahadori, Fatemeh Jaleh, Mohammad Ali Jalili, and Amaneh Mohammadi 543 Roushandeh. 2015. "Down-Regulation of the Autophagy Gene, ATG7, Protects Bone 544 Marrow-Derived Mesenchymal Stem Cells from Stressful Conditions.” Blood Research 50 545 (2): 80-86. https://doi.org/10.5045/br.2015.50.2.80.

546

547

548

549 550

Mutlu, Levent, Demetra Hufnagel, and Hugh S. Taylor. 2015. "The Endometrium as a Source of Mesenchymal Stem Cells for Regenerative Medicine1.” Biology of Reproduction 92 (6). https://doi.org/10.1095/biolreprod.114.126771.

Patel, Amit N., Craig H. Selzman, Ganesh S. Kumpati, Stephen H. McKellar, and David A. Bull. 2016. "Evaluation of Autologous Platelet Rich Plasma for Cardiac Surgery: Outcome Analysis of 2000 Patients." Journal of Cardiothoracic Surgery 11 (1). https://doi.org/10.1186/s13019-016-0452-9.

Pertschuk, Louis P. 1990. Immunocytochemistry for Steroid Receptors. CRC Press.

Pham, Liem Hieu, Ngoc Bich Vu, and Phuc Van Pham. 2019. “The Subpopulation of CD105 Negative Mesenchymal Stem Cells Show Strong Immunomodulation Capacity Compared to CD105 Positive Mesenchymal Stem Cells.” Biomedical Research and Therapy 6 (4): 313140. https://doi.org/10.15419/bmrat.v6i4.538.

Ponnaiyan, Deepa, and Visakan Jegadeesan. 2014. "Comparison of Phenotype and Differentiation Marker Gene Expression Profiles in Human Dental Pulp and Bone Marrow 
560

561

562

563

564

565

566

567

568

569

570

571

572

573

574

575

576

577

578

579

580

581

582

Mesenchymal Stem Cells.” European Journal of Dentistry 8 (3): 307-13. https://doi.org/10.4103/1305-7456.137631.

Reghini, Maria Fernanda S., Carlos Ramires Neto, Lorenzo G. Segabinazzi, Maria Manoela B. Castro Chaves, Camila de Paula F. Dell'Aqua, Maria Clara C. Bussiere, José Antonio Dell'Aqua, Frederico O. Papa, and Marco Antonio Alvarenga. 2016. “Inflammatory Response in Chronic Degenerative Endometritis Mares Treated with Platelet-Rich Plasma.” Theriogenology 86 (2): 516-22. https://doi.org/10.1016/j.theriogenology.2016.01.029.

Tanida, Isei, Takashi Ueno, and Eiki Kominami. 2008. "LC3 and Autophagy.” In Autophagosome and Phagosome, edited by Vojo Deretic, 77-88. Totowa, NJ: Humana Press. https://doi.org/10.1007/978-1-59745-157-4_4.

Tempest, Nicola, Alison Maclean, and Dharani K. Hapangama. 2018. "Endometrial Stem Cell Markers: Current Concepts and Unresolved Questions.” International Journal of Molecular Sciences. https://doi.org/10.3390/ijms19103240.

Theoret, Christine L., and Ted S. Stashak. 2014. "Integumentary System: Wound Healing, Management, and Reconstruction.” In Equine Emergencies, edited by James Orsini and Thomas Divers, Fourth Edi, 238-67. Elsevier Inc. https://doi.org/10.1016/b978-1-45570892-5.00019-2.

Wang, Xuyi, Evan R. Simpson, and Kristy A. Brown. 2015. "P53: Protection against Tumor Growth beyond Effects on Cell Cycle and Apoptosis." Cancer Research. American Association for Cancer Research Inc. https://doi.org/10.1158/0008-5472.CAN-15-0563.

Yazigi Junior, João Alberto, João Baptista Gomes dos Santos, Bruno Rodrigues Xavier, Marcela Fernandes, Sandra Gomes Valente, and Vilnei Mattiolli Leite. 2015. “Quantification of Platelets Obtained by Different Centrifugation Protocols in SHR Rats." Revista Brasileira 
de Ortopedia (English Edition) 50 (6): 729-38. https://doi.org/10.1016/j.rboe.2015.10.008.

584 Yuksel, Esra Pancar, Gokhan Sahin, Fatma Aydin, Nilgun Senturk, and Ahmet Yasar Turanli.

585 2014. "Evaluation of Effects of Platelet-Rich Plasma on Human Facial Skin.” Journal of

586 Cosmetic and Laser Therapy 16 (5): 206-8. https://doi.org/10.3109/14764172.2014.949274.

587 Yun, Seong Hoon, Eun Hye Sim, Ri Young Goh, Joo In Park, and Jin Yeong Han. 2016.

588 "Platelet Activation: The Mechanisms and Potential Biomarkers." BioMed Research

589 International. https://doi.org/10.1155/2016/9060143.

590 Zadehmodarres, Shahrzad, Saghar Salehpour, Nasrin Saharkhiz, and Leila Nazari. 2017.

591 "Treatment of Thin Endometrium with Autologous Platelet-Rich Plasma: A Pilot Study."

592 Jornal Brasileiro de Reproducao Assistida 21 (1): 54-56. https://doi.org/10.5935/1518-

$593 \quad 0557.20170013$.

594 Zhou, Shuanhu, Yoram Zilberman, Karsten Wassermann, Steven D. Bain, Yoel Sadovsky, and 595 Dan Gazit. 2001. "Estrogen Modulates Estrogen Receptor $\alpha$ and $\beta$ Expression, Osteogenic 596 Activity, and Apoptosis in Mesenchymal Stem Cells (MSCs) of Osteoporotic Mice.”

597 Journal of Cellular Biochemistry 81: 144-55. https://doi.org/10.1002/jcb.1096.

598 


\section{Figure 1}

Characterization of the mesenchymal cell cultures isolated from rat uterus.

Flow cytometry analysis: (a) representative forward and side scattering dot plot with the region of interest (R1). The percentages of $C D 90, C D 105, C D 45$, and CD34 positive cells in $R 1$ are indicated (b). Green curve corresponds to the control (stained with secondary antibodies only). Anti-vimentin staining (c): upper image - negative control (secondary antibodies only); lower image - immunocytochemistry with anti-vimentin antibodies (red) and the nuclei counterstained with DAPI (blue). Bars, $20 \mu \mathrm{m}$. Induced cell differentiation assay (d): adipogenic differentiation revealed by Sudan III staining, osteogenic differentiation revealed by alizarin red staining, and chondrogenic differentiation revealed by alcian blue staining. Upper panel - non-induced control cells, bottom panel - cells following the induction of differentiation. Bars, $50 \mu \mathrm{m}$. One experimental block is marked by a dotted line. 

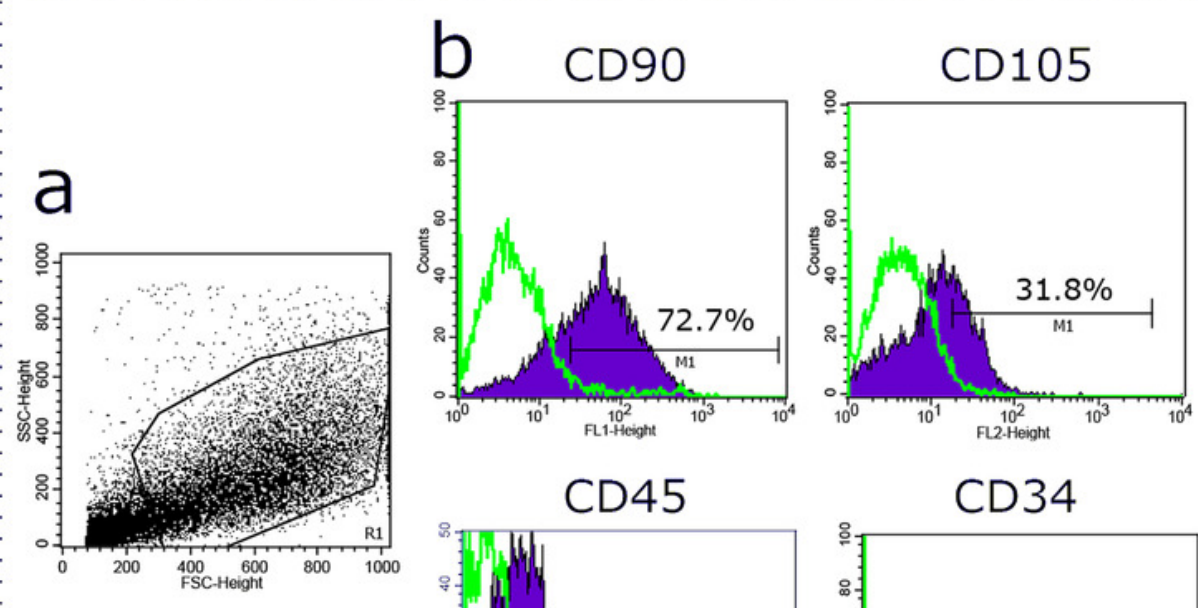

C
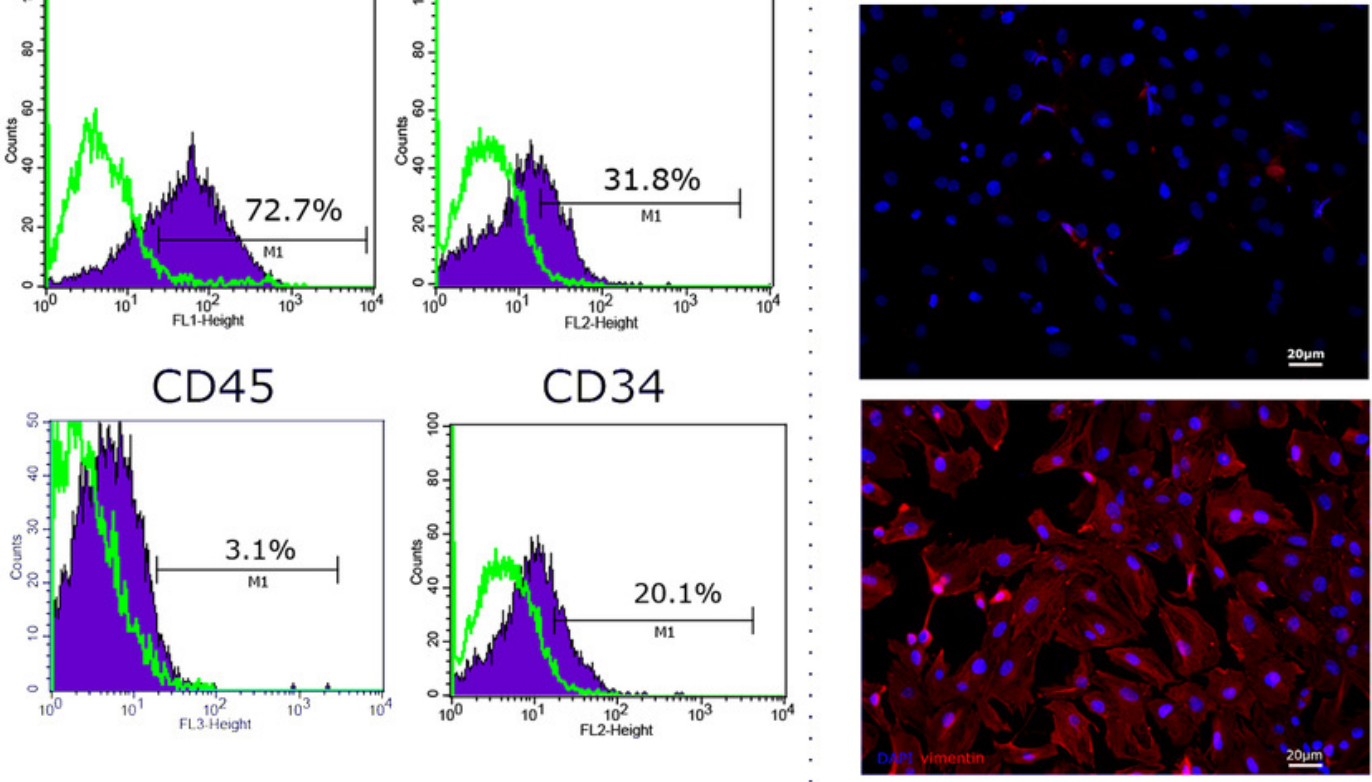

d

Adipogenic

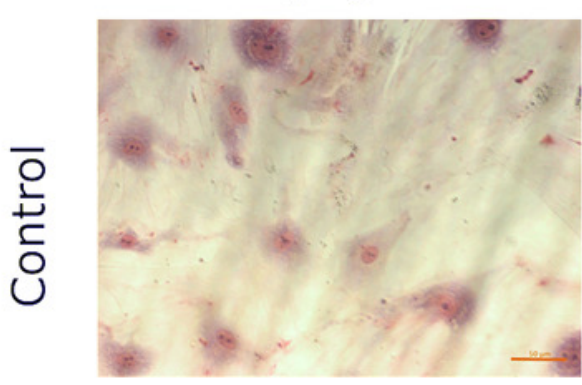

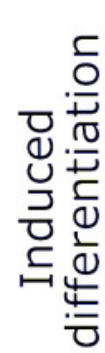

Osteogenic
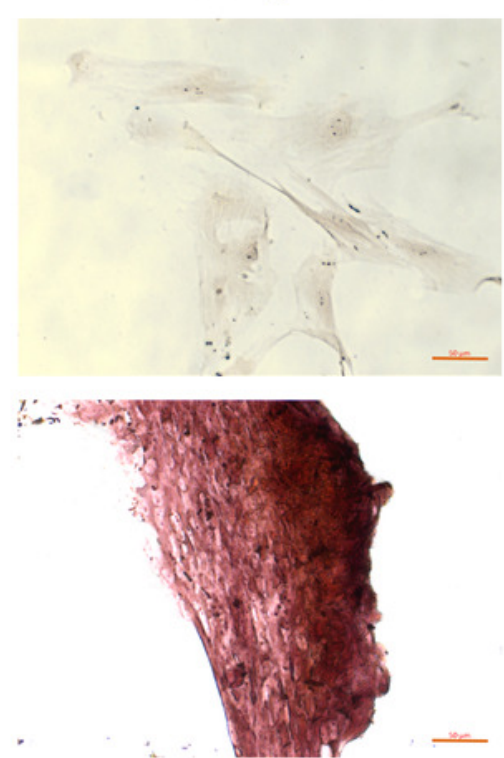

Chondrogenic
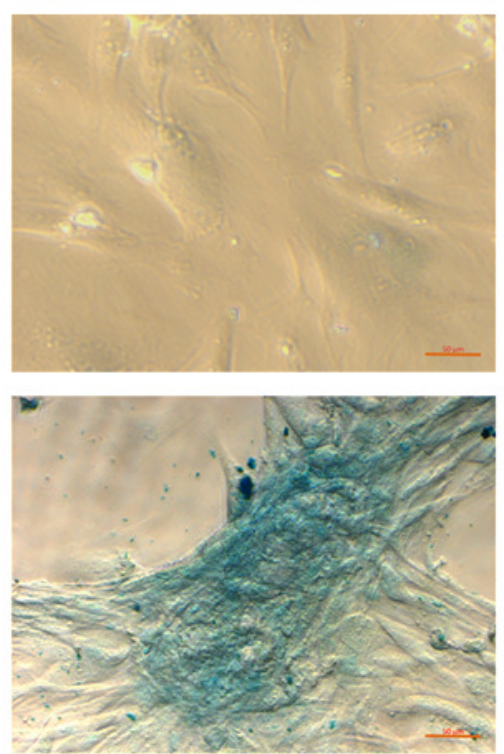


\section{Figure 2}

Assessment of cell viability and cell death following the $24 \mathrm{~h}$ incubation of rat uterine mesenchymal stem/stromal cells (MSCs) with complete growth medium (CGM), plateletrich plasma (PRP), or ordinary plasma (OP).

Immunocytochemical staining (a) for Ki-67 (green) after $24 \mathrm{~h}$ exposure of the MSC cultures to CGM (left), PRP (central), and OP (right); the nuclei were counterstained with DAPI (blue). Bars, $20 \mu \mathrm{m}$. (b) Proliferation index calculated as the number of Ki-67 positive nuclei divided by the total number of nuclei. * $p<0.05$ vs CGM. (c) Representative western blot membranes with the proteins isolated from MSCs after $24 \mathrm{~h}$ incubation with the studied agents, stained with p53, BCl-2, LC3B, and GAPDH specific antibodies. Relative protein levels of p53 (d), BCl-2 (e), and LC3B (f), normalized by GAPDH level. * $p<0.05$ vs CGM group. One experimental block is marked by a dotted line. 


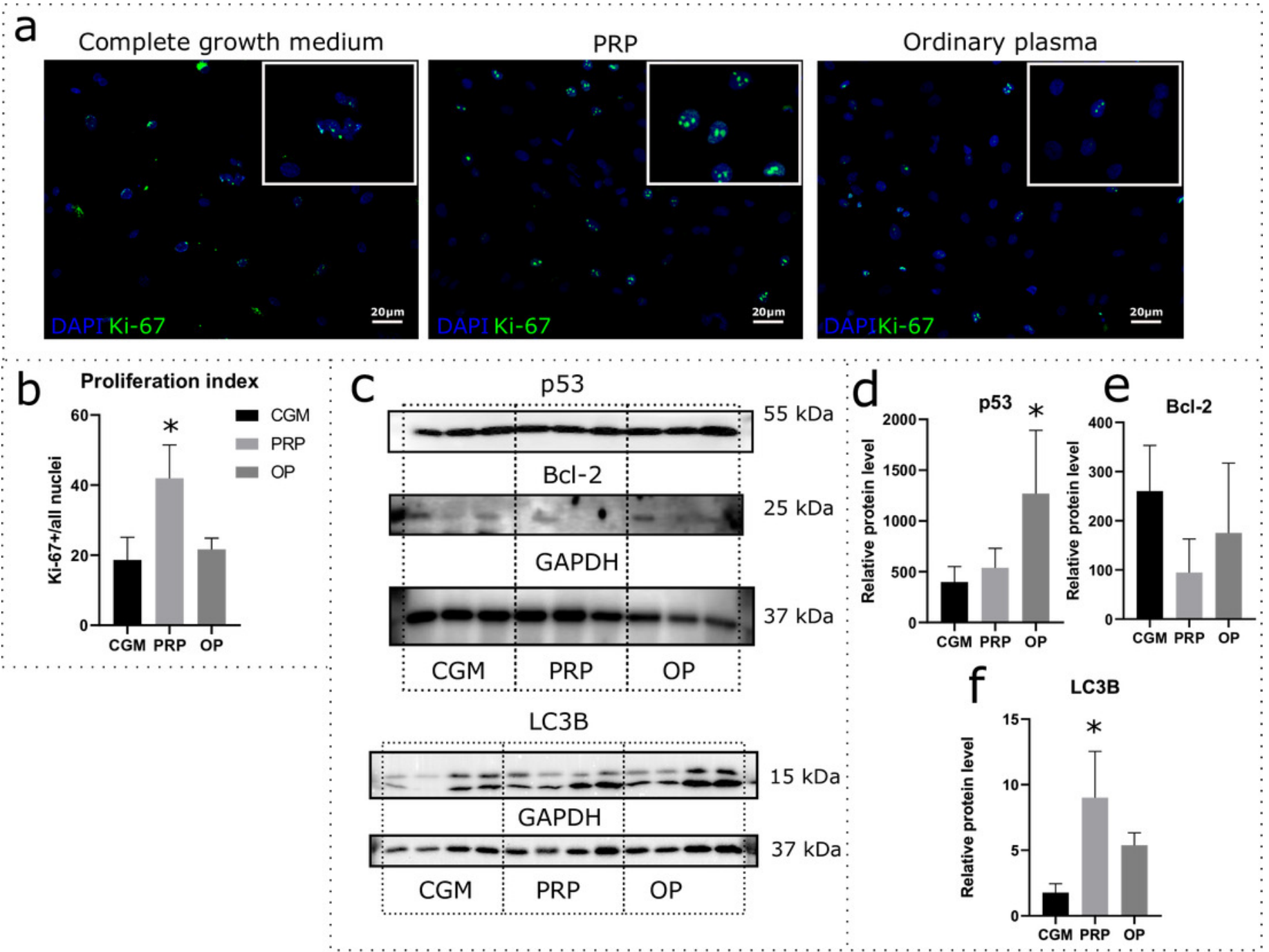


Figure 3

Western blot membrane stained with antibodies to matrix metalloproteinase 9 (MMP9) and estrogen receptor $\alpha(E R \alpha)$.

A representative western blot membrane with the proteins isolated from mesenchymal stem/stromal cells after $24 \mathrm{~h}$ incubation with complete growth medium (CGM), platelet-rich plasma (PRP), or ordinary plasma (OP), stained with MMP9 (a), ER $\alpha$ (c), and GAPDH specific antibodies. Relative protein levels of MMP9 (b) and ER $\alpha(d)$, normalized by GAPDH level. * $p<$ 0.05 vs CGM group.

a

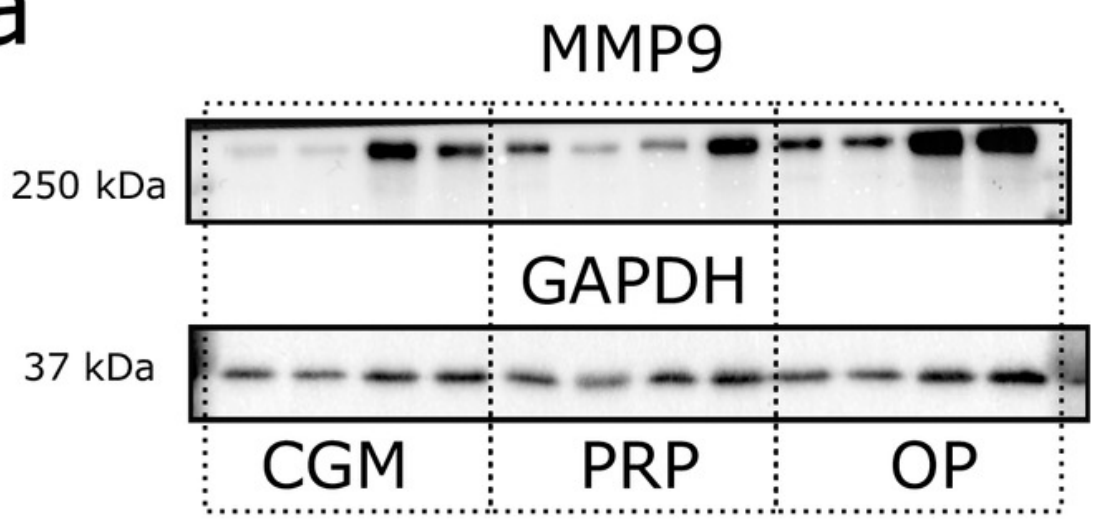

C

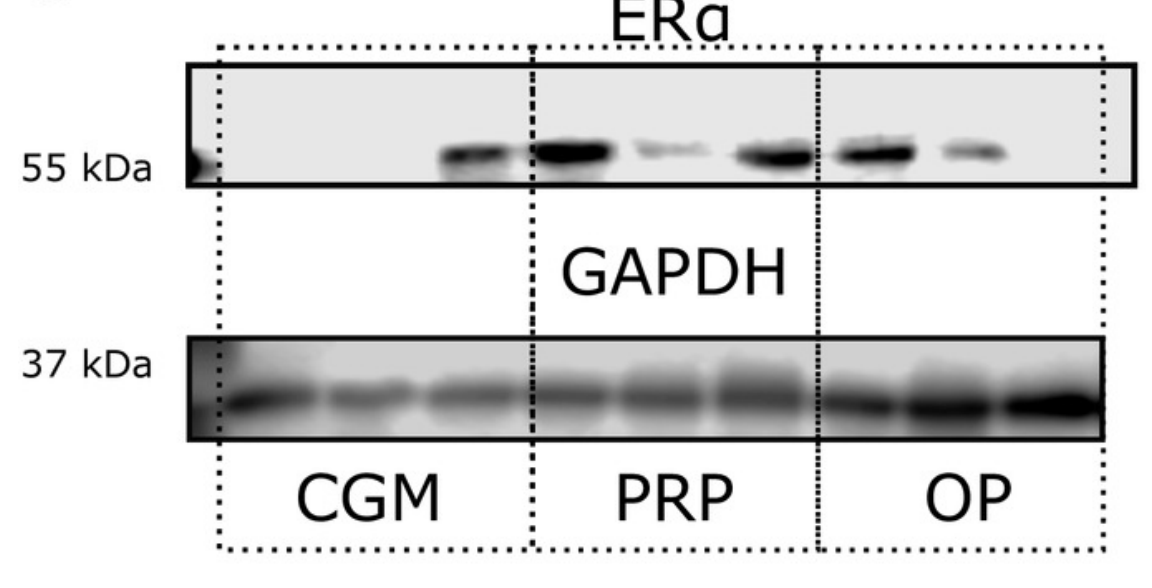

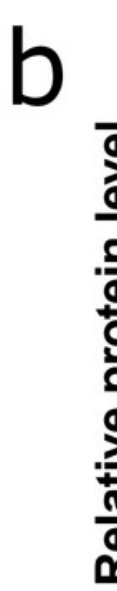

d
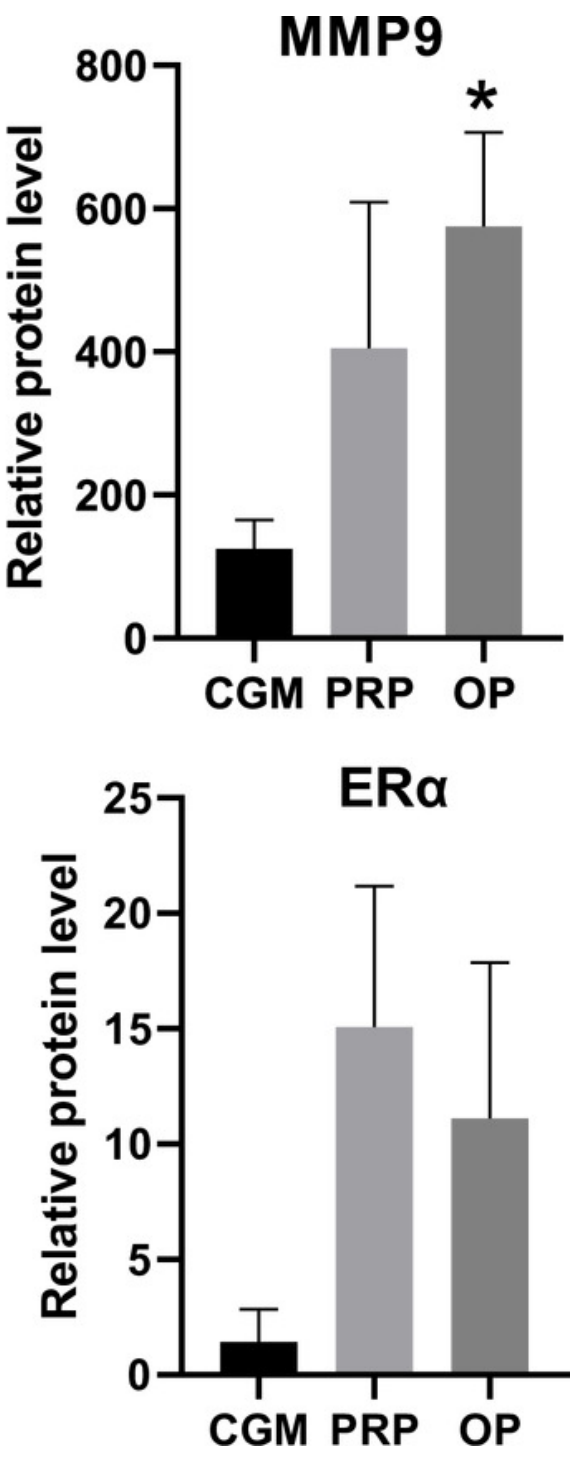